\section{REFERENCIAS}

1. United Nations. The Millennium Development Goals Report 2013 New York: United Nations; 2013. [Acceso: 10 febrero de 2018]. Disponible en: http:/ / www.un.org / millenniumgoals / pdf / report-2013/mdg-report-2013english.pdf.

2. Blencowe $\mathrm{H}, \operatorname{Vos} \mathrm{T}$, Lee $\mathrm{AC}$, et al. Estimates of neonatal morbidities and disabilities at regional and global level for 2010: introduction, methods overview and relevant findings from the Global Burden of Disease study. Pediatr Res 2013; 74(Suppl 1):4-16.

3. Murray C, Vos T, Lozano R, et al. Disability-adjusted life years (DALYs) for 291 diseases and injuries in 21 regions, 1990-2010: a systematic analysis for the Global Burden of Disease Study 2010. Lancet 2012; 380(9859):2197-223.

4. Brisson M, García Conde S, Di Pietro L. Objetivos de Desarrollo del Milenio. La Cumbre del Milenio y los compromisos internacionales. Buenos Aires: Consejo Nacional de Coordinación de Políticas Sociales; 2014. [Acceso: 10 de noviembre de 2017]. Disponible en: http:/ / www.odsargentina.gob.ar/public/documentos/seccion_ publicaciones/odm/la_cumbre_del_milenio_y_los_ compromisos_internacionales.pdf.

5. Lawn J, Blencowe H, OzaS, et al. Every Newborn: progress, priorities, and potential beyond survival. Lancet 2014; 384(9938):189-205.

6. Finkelstein J, Duhau M, Speranza A. Evolución de la mortalidad infantil en Argentina en el marco de los Objetivos de Desarrollo del Milenio. Arch Argent Pediatr 2016; 114(3):216-22.

7. Lawn J, Blencowe H, Pattinson R, et al. Stillbirths: Where? When? Why? How to make the data count? Lancet 2011; 377(9775):1448-63.

8. Grandi C, Nascente L, Cardoso V. Impactodela mortalidad fetal sobrela prematurez en Argentina: estudio poblacional. Rev Fac Cien Med 2017; 74(4).

9. Barker DJ. The developmental origins of chronic adult disease. Acta Paediatr Suppl 2004; 93(446):26-33.

10. Blencowe H, Cousens S, Oestergaard MZ, et al. National, regional, and worldwide estimates of preterm birth rates in the year 2010 with time trends since 1990 for selected countries: a systematic analysis and implications. Lancet 2012;379(9832):2162-72.

\title{
Publicaciones predadoras Predatory journals
}

Es muy frecuente que los lectores de estas líneas hayan recibido mensajes para invitarlos insistentemente a enviar un artículo para publicar en alguna revista científica. En ocasiones, ensalzan las características de una publicación absolutamente ignota; tampoco es infrecuente que apelen a su vanidad resaltando su supuesta pericia en el tema.

Son comunes mensajes como este: "Estimado profesor .............., hemos leído su artículo titulado Prevalencia de la publicación predatoria en el mundo. Impresionados por la calidad de su trabajo, lo contactamos para pedirle que nos envíe su próximo estudio sobre el tema". También es habitual que, a través de la misma modalidad, se los invite a formar parte de comités editoriales de revistas que tienen nombres "parecidos" a reconocidas publicaciones científicas.

¿Qué se esconde detrás de todo esto? Este tipo de mensajes forma parte, en su mayoría, del llamado mundo de las "publicaciones predadoras" (predatory journals), cuyo objetivo principal es obtener dinero, de manera poco ética, aunque no ilegal. Estas publicaciones tienen poco o ningún cuidado con la calidad de los trabajos que publican, no siguen los estándares de buenas prácticas en publicación académica e imponen un arancel al autor, quien financia la publicación de su trabajo. ${ }^{1}$

El término predatory journals fue acuñado en 2010 por Jeffrey Beall, un bibliotecario de la Universidad de Colorado, quien creó un blog en el que publicó una lista de las editoriales y revistas que desarrollaban esta práctica. La controversia que suscitó esta publicación incluyó comentarios editoriales y demandas judiciales en ambos sentidos, que finalizó con el "levantamiento" de su blog en $2017 .{ }^{2}$

La práctica habitual de las publicaciones predadoras incluye características como las siguientes: ${ }^{3}$

- Promesa de una publicación en tiempo breve.

- Anuncio de un factor de impacto falso.

- Falta de claridad sobre el arancel por evaluación.

- Falta de transparencia sobre la localización de sus oficinas.

- Utilización de nombres parecidos a los de publicaciones prestigiosas.

- Comunicación masiva por spam.

¿Cómo empezó todo esto?

Muchos centran el inicio del problema en la iniciativa Open Access. ${ }^{4}$ La misma surgió a partir de la conjunción de varios factores: por 
un lado, la presión de "publicar o morir" en el ámbito académico ha hecho que cada vez hubiera más manuscritos para un número limitado de publicaciones dispuestas a recibirlos. Por otro lado, el advenimiento de internet con la consiguiente modificación del negocio editorial, la globalización del conocimiento y la presión por el acceso libre a él llevaron a cuestionar la limitación de la información científica que imponían los grandes grupos editores. ${ }^{5}$ Finalmente, el gran número de publicaciones a las que hay que acceder para mantenerse actualizado hace económicamente inviable la suscripción a todas ellas, tanto para un individuo como para las grandes instituciones.

La iniciativa Open Access busca el acceso libre a la información científica disponible en internet. Como la publicación científica tiene un insoslayable costo que no sería cubierto por la suscripción de los lectores, se sugirió que los autores se hicieran cargo de la publicación pagando lo que se conoce como "cargo por procesar el artículo" (article processing fee). Este procesamiento incluye los controles que una buena publicación debe tener para evitar aquellas sin mérito científico o claramente fraudulentas. Lamentablemente, esta iniciativa altruista dio lugar a grupos con pocos escrúpulos que solo perseguían rédito económico: las publicaciones predadoras. Ellas difunden los manuscritos a cambio del cargo por procesamiento, pero el ansia de lucro y la falta de controles editoriales eficaces los lleva a publicar investigaciones de muy baja calidad o aun fraudulentas.

\section{¿Cuál es el resultado de esta práctica? ${ }^{1}$}

Aquellos que, incluso luego de estas advertencias, decidan acercarse al mundo de las publicaciones predatorias deben saber cuáles son los riesgos. Sus artículos no recibirán el aporte de una buena revisión por pares, práctica que reconocidamente mejora la calidad científica de un manuscrito. Además, sus publicaciones pueden desaparecer de internet después de un tiempo, conociendo la inestabilidad de estas compañías. Sus trabajos pueden ser difíciles de localizar por no hallarse la publicación indexada en alguna base de datos seria. Finalmente, puede resultar humillante encontrar su artículo asociado a la desconfianza que genera una publicación predadora, cuya falta de seriedad ya ha sido desenmascarada. ${ }^{6,7}$

Todo el respaldo que tiene una publicación científica es su prestigio, ganado sobre la base de una trayectoria con estricto control del material que se publica, rigor científico y honestidad intelectual. Ese prestigio respalda cada una de las investigaciones publicadas y permite que tanto sus autores como los lectores confíen en la solidez académica de los contenidos.

Verónica Aguerre

Hospital de Pediatría SAMIC “Prof. Dr. Juan P. Garrahan”

Fernando Ferrero

Hospital General de Niños Pedro de Elizalde

http:/ / dx.doi.org/10.5546/aap.2018.240

Texto completo en inglés:

http:/ / dx.doi.org/10.5546/ aap.2018.eng.240

Cómo citar: Aguerre V, Ferrero F. Publicaciones predadoras. Arch Argent Pediatr 2018;116(4):240-241.

\section{REFERENCIAS}

1. O'Donell M. Understanding Predatory Publishers. Library guides. Iowa State University. [Acceso: 20 de marzo de 2018]. Disponibleen:http:/ / instr.iastate.libguides.com/ predatory.

2. Beall J. What I learned from predatory publishers. Biochem Med (Zagreb) 2017; 27(2):273-8.

3. Beall J. Best practices for scholarly authors in the age of predatory journals. Ann R Coll Surg Engl 2016; 98(2):77-9.

4. Björk BC, Welling P, Laakso M, et al. Open Access to the Scientific Journal Literature: Situation 2009. PLoS One 2010; 5(6):e11273.

5. Monbiot G. Academic publishers make Murdoch look like a socialist. The Guardian. London: 2012 August 29. [Acceso: 20 demarzo de2018].Disponibleen: https: / / www.theguardian. com/ commentisfree/2011/aug/29/academic-publishersmurdoch-socialist.

6. Sorokowski P, Kulczycki E, Sorokowska A, et al. Predatory journals recruit fake editor. Nature 2017; 543(7646):481-3.

7. Bohannon J. Who's afraid of peer review? Science 2013; 342(6154):60-5. 